\title{
Fragmentation dynamics of molecular hydrogen in strong ultrashort laser pulses
}

\author{
A. Rudenko, B. Feuerstein, K. Zrost, V.L.B. de Jesus*, T. Ergler, \\ C.D. Schröter, R. Moshammer, and J. Ullrich \\ Max-Planck-Institut für Kernphysik, D-69029 Heidelberg, Germany \\ * present address: Centro Federal de Educação Tecnológica de Química de Nilópolis/RJ, \\ Rua Lucio Tavares 1045, Centro - Nilópolis - 26530-060, Rio de Janeiro, Brazil
}

We present the results of a systematic experimental study of dissociation and Coulomb explosion of molecular hydrogen induced by intense ultrashort (7-25 fs) laser pulses. Using coincident recoil-ion momentum spectroscopy we can distinguish the contributions from dissociation and double ionization even if they result in the same kinetic energies of the fragments. The dynamics of all fragmentation channels drastically depends on the pulse duration and for 7 fs pulses becomes extremely sensitive to the pulse shape.

32.80.Rm, 31.90.+s, 32.80.Fb

\section{Introduction}

The fragmentation and ionization of the two simplest molecules $\mathrm{H}_{2}$ and $\mathrm{H}_{2}{ }^{+}$(or alternatively $\mathrm{D}_{2} / \mathrm{D}_{2}^{+}$) in intense optical fields has been extensively studied both theoretically and experimentally. In contrast to the atomic case, the molecule exhibits three timescales, the fast electronic motion (as scale), the vibrational (fs scale) and rotational (ps scale) nuclear motion. The vibrational degree of freedom is of particular interest since its timescale matches the duration of state-of-the-art optical laser pulses. 
Considerable theoretical work was done for the neutral hydrogen molecule (see, e.g. Yu et al 1996, Walsh et al 1998, Harumiya et al 2002, Saenz 2002a,b) and for the molecular ion (see, e.g. Zuo and Bandrauk 1995, Chelkovski et al 1996,1999, Numico et al 1997, Dundas et al 2000, Bandrauk and Shon 2002, Feuerstein and Thumm 2003). Some specific properties make the latter a model laboratory system for lasermatter interactions. First, there exists only one bound potential curve, i.e. the electronic ground state - all other electronically excited states are purely repulsive and converge towards the Coulombic $1 / R$ curve, where $R$ is the internuclear distance (see Figure 1). Second, the degeneracy of the two lowest electronic states $1 \mathrm{~s} \sigma_{\mathrm{g}}$ and $2 \mathrm{p} \sigma_{\mathrm{u}}$ lead to a strong dipole interaction of these levels in the presence of external electrical fields which is essential for the coupling of the system to laser radiation. Third, the quite large difference in the ionization energies of $\mathrm{H}_{2}(15.2 \mathrm{eV})$ and $\mathrm{H}_{2}{ }^{+}(29.9 \mathrm{eV})$ is the basis of a two-step treatment: Starting with the neutral molecule in a strong laser pulse the first electron will be easily removed creating the ionic state which will subsequently undergo further fragmentation processes. Most experiments have used the neutral molecule as a target (Zavriyev et al 1993, Walsh et al 1997, Thomson et al 1997, Gibson et al 1997, Frasinski et al 1999, Posthumus et al 2000, Rottke et al 2002). However, the formation and fragmentation of $\mathrm{H}_{2}{ }^{+}$occurs in the same laser pulse and, thus, cannot be completely disentangled (Walsh et al 1998).

Hence, there is a growing interest in using molecular ions as targets (Williams et al 2000, Sändig et al 2000, Shellhammer et al 2000) or in getting better insight into the time dependence of the fragmentation dynamics. The latter is not at last stimulated by the fact that in contrast to the ionic case using a neutral molecule as a target opens an additional possibility to probe the dynamics of the nuclar motion. Recently the pioneering concept of a "molecular clock" (Niikura et al 2002,2003, Alnaser et al 
2003) was introduced. The idea is based on the so-called recollision model (Corkum 1993). Here, one considers the combined propagation of the nuclear and electronic wavepackets, which is started by the first ionization step. The emitted electron is driven back and forth by the oscillating laser field and may recollide after a welldefined time with its parent ion while the nuclei have moved apart according to their motion on the new $\mathrm{H}_{2}{ }^{+}$potential curve. Emission of the second electron induced by

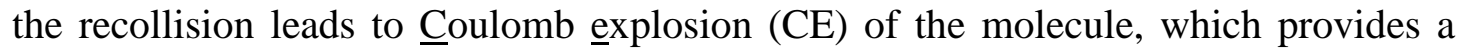
mapping of the nuclear wavepacket at the time of recollision via the relation of the kinetic energy release (KER) of the fragments $E_{\text {kin }}=1 / R$. However, to read the "molecular clock" one has to know via which channel the fragmentation takes place. This requires a detailed and comprehensive modelling of the recollision process. Very recently, good agreement of theory and experiment has been achieved (Alnaser et al 2003, Tong et al 2003). However, there are still open questions remaining. In particular, there is an ongoing debate whether recollision-induced dissociation contributes to the fragments detected in the "molecular clock" scheme.

The present work focuses on the influence of the temporal structure of short and ultrashort $\left(<10 \mathrm{fs}\right.$ ) laser pulses on the fragmentation dynamics of $\mathrm{H}_{2}$ and on the problem of reliable distinguishing between the contributions from the dissociation and CE for the cases where they lead to fragments with similar kinetic energies. The paper is structured as follows: In Section 2 a brief description of the experimental set-up is given, followed by an overview of the various fragmentation channels and the driving mechanisms in section 3. The experimental results are presented and discussed in Section 4, and conclusions are given in Section 5. 


\section{Experimental setup}

The measurements were performed using a new "reaction microscope" (Ullrich et al 2003) designed to meet the specific requirement of the experiments with highintensity lasers (see de Jesus et al 2004a for details). We used linearly polarized radiation of a Kerr-lens mode locked Ti:sapphire laser at $795 \mathrm{~nm}$ wavelength with 25 fs pulse width (FWHM). To generate few-cycle pulses they were spectrally broadened in a gas-filled hollow fiber and then compressed to 7 fs (FWHM) by chirped mirrors and a prism compressor. Fluctuations of the laser intensity from pulse to pulse were monitored during the experiment and did not exceed 5\%. Absolute calibration of the peak intensity was performed using a clear kink in the measured photoelectron momentum distribution, which corresponds to the maximum drift momentum of $2 \sqrt{U_{p}}$ that electrons can gain from the laser field (de Jesus et al 2004b).

The laser beam was focused to a spot size of $\sim 7 \mu \mathrm{m}$ on the collimated supersonic gas jet in the ultra-high vacuum chamber $\left(2 \cdot 10^{-11} \mathrm{mbar}\right)$. Charged reaction fragments were guided to two position-sensitive channel plate detectors by weak electric (2 V/cm) and magnetic $(7 \mathrm{G})$ fields applied along the laser polarization axis. From the time-of-flight and position on the detectors the full momentum vectors of the recoil ions and electrons were calculated.

A typical time-of-flight (TOF) spectrum of $\mathrm{H}_{2}$ fragmentation by 25 fs laser pulse is shown in Figure 2a. The most prominent line at the right side of the spectrum corresponds to $\mathrm{H}_{2}^{+}$ions. $\mathrm{H}^{+}$fragments form two groups of peaks nearly mirror symmetric with respect to the full vertical line indicating the TOF of the protons with zero longitudinal (along the polarization direction) momentum. 'Forward' protons are those going directly to the ion detector, while 'backward' ones go first to the opposite hemisphere and then are turned back by the extraction field. Thus, the $\mathrm{H}^{+}$peaks 
corresponding to the various fragmentation channels appear twofold in the spectrum. Slight asymmetry in the peaks position is due to the fact that the longitudinal momentum is not exactly linear with TOF. Here it should be noted that the heights of the peaks, i.e. the branching ratios of the different channels depend on the acceptance of the spectrometer, which differs for different fragment energies (see de Jesus et al 2004a for details). Thus only the qualitative changes of these ratios with intensity and pulse duration will be considered here.

Figure $2 \mathrm{~b}$ shows corresponding energy spectrum for the 'forward' protons, where the same set of peaks can be seen. Fragmentation mechanisms giving rise to these structures are discussed in the next section.

\section{Fragmentation channels of $\mathrm{H}_{2}$ in strong $800 \mathrm{~nm}$ laser pulses}

Figure 1 shows the different ionization and fragmentation pathways of $\mathrm{H}_{2}$ in a strong $800 \mathrm{~nm}$ laser field in form of a potential curve diagram. By single ionization of $\mathrm{H}_{2}$ the initial vibrational ground state is promoted in a vertical transition onto the electronic ground state potential curve $1 \mathrm{~s} \sigma_{\mathrm{g}}$ of $\mathrm{H}_{2}{ }^{+}$. Since the wave packet is not an eigenstate anymore it will start to propagate on the new potential curve defining a timescale for subsequent fragmentation processes. The molecular ion can either dissociate or be further ionized leading to a pair of protons (Coulomb explosion). Up to now there were no experimental evidences found for direct molecular dissociation leading to a pair of neutral hydrogen atoms (Posthumus 2004).

\subsection{Dissociation}

Dissociation is driven by a strong dipole interaction of the two lowest electronic states $1 \mathrm{~s} \sigma_{\mathrm{g}}$ and $2 \mathrm{p} \sigma_{\mathrm{u}}$ in the presence of a strong field along the molecular axis. In a cycle- 
averaged Floquet description (Shirley 1965, Bandrauk and Sink 1981) one finds a series of dressed potential curves spaced by the photon energy. Via avoided crossings the molecular ion can dissociate by a net absorption of one or two photons. In the latter case, absorption of three photons in the first avoided crossing is followed by a re-emission of one photon at the second crossing. Since for each photon the parity of the molecular state must change, there is no direct two-photon process. In the fragment kinetic energy spectrum (see Figure 2b) these channels manifest themselves as two peaks below $1 \mathrm{eV}$ per proton ( $1 \omega$ and $2 \omega$ peaks). A comprehensive discussion on the dynamics and interplay of different dissociation mechanisms referred to as "bond softening" (Bucksbaum et al 1990), vibrational trapping, or "bond-hardening" (Frasinsky et al 1999), “above-threshold” (Giusti-Suzor et al 1990), “belowthreshold" (Numico et al 1997) or "zero-photon" (Posthumus et al 2000) dissociation can be found in the reviews of Giusti-Suzor et al (1995) and Posthumus (2004).

\subsection{Sequential double ionisation (SDI)}

The mechanism of SDI in strong laser fields, which is well known for atoms, becomes more complex in the molecular case since the ionisation potential of the molecular ion depends on the internuclear separation $R$. In addition, at intermediate $R$ an even stronger enhancement of the field ionisation rates is observed due to the interaction of the $1 \mathrm{~s} \sigma_{\mathrm{g}}$ and $2 \mathrm{p} \sigma_{\mathrm{u}}$ states, which causes an oscillating localisation of the electron cloud at one of the nuclei. This effect of charge resonance enhanced ionisation (CREI) gives the largest ionisation probabilities for $\mathrm{H}_{2}$ in a range of internuclear distance from 3.5 to 7 a.u. corresponding to a KER per proton of 2 to $4 \mathrm{eV}$ (Figure 2b). Since the dipole interaction occurs along the molecular axis, the corresponding $\mathrm{CE}$ contribution is strongly peaked along the laser polarization direction (Posthumus 2004). CREI was 
found to be a dominant SDI channel in most of the measurements on $\mathrm{H}_{2}$ and $\mathrm{D}_{2}$. However, the results of recent experiments (Legare et al. 2003, Osipov et al 2004) have shown that if few-cycle laser pulses are used, the "atomic-like" SDI, which results in more energetic CE fragments with more isotropic angular distribution, starts to compete with CREI. In (Legare et al. 2003) these two channels were referred to as "sequential ionization" and "enhanced ionization". This should not lead to confusion: both processes are definitely sequential and the essential difference is the extreme Rdependence of the "enhanced ionization" (CREI).

\subsection{Non-sequential double ionisation (NSDI)}

In the case of a linearly polarized laser field the first emitted electron can be driven back and recollide after about three quarters of an optical cycle with its parent ion. The returning electron may either kick out directly the second electron through recollision-ionization (RI) or promote the bound electron into an excited state, which can then be easily field ionised. This mechanism of recollision-excitation followed by subsequent field ionisation (RESI) was found to play a decisive role for NSDI of atoms (Feuerstein et al 2001, de Jesus et al 2004a,b). For $\mathrm{H}_{2}$ the NSDI channel has been identified as a high-energy shoulder in the fragment energy spectra for linear polarization, which disappears in the circular case as shown in Figure $2 \mathrm{~b}$ (see also Staudte et al 2002, Sakai et al 2003, Legare et al 2003). NSDI leads to the largest fragment energies since it is a fast process, which for the first electron return gives the nuclei less than one optical cycle to separate. In a recent work based on the "molecular clock" principle mentioned above it was shown by comparison of experimental and theoretical results that RESI is the dominating NSDI channel (Alnaser et al 2003, Tong et al 2003). 


\section{Results and discussion}

\subsection{From short to ultrashort pulses: Influence of the pulse shape}

Figure 3 shows the evolution of the time-of-flight (TOF) spectra of the $\mathrm{H}_{2}$ fragments with the decreasing pulse duration. The spectra for 25 fs pulses at peak intensities of 0.2, 0.3 and $0.5 \mathrm{PW} / \mathrm{cm}^{2}$ are shown in figure 3a. Similar to Figure 2a, with lowest kinetic energy we find the $1 \omega$ and $2 \omega$ dissociation peaks followed by the broad structure due to CREI. As the intensity is increased the branching ratio of the $1 \omega \mathrm{vs}$. $2 \omega$ dissociation becomes smaller and CE starts to compete with the dissociation channel. A similar intensity dependence of the spectra was observed in previous experiments (Thomson et al 1997, Staudte et al 2002). If we now reduce the pulse duration while keeping the peak intensity approximately fixed the fragmentation pattern changes significantly (Figure $3 \mathrm{~b}$ : $10 \mathrm{fs}$, Figure $3 \mathrm{c}$ : $7 \mathrm{fs}$ ). We find the branching ratio of $1 \omega$ and $2 \omega$ dissociation channels reversed and the corresponding peaks broadened, a feature that will be discussed in section 4.3. With the decreasing pulse duration we observe a strong suppression of the CE (CREI) channel compared to dissociation. Intuitively it is apprehensible: when the pulse length becomes shorter than the vibrational period, the pulse may be gone before the molecule extends to the critical internuclear distances corresponding to the region of enhanced ionization.

Figure $3 \mathrm{c}$ depicts two spectra obtained with the pulses of the same temporal width (FWHM) (7 fs) but different shapes. Figure 4 shows the measured frequency spectra (, the Fourier limit for the intensity profile and measured $2^{\text {nd }}$ order autocorrelation for both pulses. Autocorrelation patterns calculated from the spectra are very close to the measured ones. Panels (a)-(c) and (d)-(f) in Figure 4 correspond to the curves 1 and 2 in Figure 3c, respectively. For the curve 1, similar to the results of Legare et al (2003) 
on $\mathrm{D}_{2}$, we find a splitting of the CE peak into two components, one corresponding to CREI, and one with higher energies, whereas for the curve 2 only the outer peak can be observed. In order to elucidate this effect in more detail, we consider the correlated longitudinal (along the field polarisation direction) momenta of two protons detected in coincidence as shown in two-dimensional diagrams in Figure 5 for 25 fs (a) and 7 fs (b) pulse width, respectively. The true coincidences are found close to the diagonal reflecting almost zero sum momentum. The remaining width is due to the momentum defect carried away by the electron which is not shown here (see de Jesus et al 2004a). All other events are due to random coincidences, mainly with protons from dissociation as it can be seen from the projected momentum spectra on the top and on the left side. In Figure 5a CREI events are found in the broad maximum on this diagonal whereas the events due to recollision form the tail extending to larger momenta. The small peak around \pm 9 a.u. consists mainly of false events due to the crossing of two bands of random coincidences. The correlation diagram for the $7 \mathrm{fs}$ pulse, which belongs to the curve 1 of Figure 3c, reveals again the disappearance of the broad CREI peak and, instead, two new peaks in its flanking parts. At lower momenta ( \pm 14 a.u.) a third peak shows up, which is certainly not an artefact since it does not fit to a band of random coincidences. Assuming all proton energy to be due to the Coulomb explosion the internuclear distance $R$ at the time when the second electron was removed can be extracted from the peak positions. Here, we find $R=3.2$ a.u. and $R=5.7$ a.u. for the two peaks visible in Figure 3c. The third peak corresponds to $R=9.4$ a.u. and, hence, the question arises how the nuclear wave packet can reach that distance within the duration of the pulse. This leads us back to Figure $3 \mathrm{c}$ and the dependence on the pulse shape. 
As it can be seen from Figure 4, for those cases where we find in the correlation spectrum a clear splitting of SDI peak the pulse shape was affected by quite strong pre and post pulses. By optimizing the pulse shape, i.e. suppressing the pre and post pulses (Figure 4d-f) two low-energy peaks are suppressed and only the peak with the highest kinetic energy (and consequently shortest delay between removal of the first and second electron) survives (curve 2 in Figure 3c). This proves that the pre and post pulses were strong enough for a subsequent ionization on a time scale of 15 to 25 fs which is much longer than the FWHM of the main peak of the temporal pulse profile. This critical dependence on the pulse shape is due to the from the fact that the molecular ion becomes more and more sensitive to field ionization as it expands with time and reaches the region of enhanced ionization where even a weak post pulse can remove the second electron. From a more technical point of view, this behaviour makes $\mathrm{H}_{2}$ a sensitive probe for the quality of ultrashort laser pulses which could be used as a diagnostic tool.

\subsection{Sequential double ionization}

The intensity dependence of the proton energy spectra for ultrashort pulses with a "clean" temporal profile, i.e. without significant pre and post pulses, is depicted in Figure 6. Here only the most energetic SDI peak, where the second ionization step occurs before the internuclear distance reaches the region of enhanced ionization, can be resolved. However, some residual structures on the position of two low-energy double ionization peaks still can be observed in the coincidence diagrams. This is more pronounced for the highest intensities: indeed, for a peak intensity of $\mathrm{PW} / \mathrm{cm}^{2}$ the post pulses on a level as low as $1 \%$ can still result in a considerable amount of enhanced ionization events. 
Over the peak intensity range from 0.2 to $2.0 \mathrm{PW} / \mathrm{cm}^{2} \mathrm{SDI}$ starts from almost negligible contribution at the lowest power density and becomes finally comparable with the dissociation channel. The maximum of the SDI peak shifts from $4.1 \mathrm{eV}$ for $0.2 \mathrm{PW} / \mathrm{cm}^{2}$ to $5.2 \mathrm{eV}$ for $2.0 \mathrm{PW} / \mathrm{cm}^{2}$ which corresponds to an internuclear separation of $R=3.3$ a.u. and $R=2.6$ a.u., respectively. This effect has been recently demonstrated theoretically by Tong and Lin (2004). In their simulations for $\mathrm{D}_{2}$ the peak positions of $\sim 6.2 \mathrm{eV}$ at $3.2 \mathrm{PW} / \mathrm{cm}^{2}$ and $\sim 5.7 \mathrm{eV}$ at $2.4 \mathrm{PW} / \mathrm{cm}^{2}$ are predicted, which is in good qualitative agreement with our results. This shift is due to the fact that for the same pulse duration at higher peak intensities the second ionization step can occur earlier, at smaller values of $R$ and thus resulting in larger Coulomb energies of the fragments. The effect is less pronounced in the low intensity regime, most likely due to a stronger R-dependence of the ionization probability (Plummer and McCann 1996). A similar shift is observed for the CREI peak (Figure 3a, see also Walsh et al 1997).

\subsection{Recollision-induced double ionization}

For NSDI of atoms by strong linearly polarized fields the electron correlation is partly reflected by the sum momentum of the electrons parallel to the laser polarization direction. In the case of an atom this is identical to the negative recoil momentum of the doubly charged ion. It is known that the ion momentum distributions along the field polarization direction for NSDI are significantly broader than for SDI (Weber et al 2000). It can even exhibit clear maxima at nonzero momenta ("double-hump" structure) in the case of a dominance of RI as, e.g. for Ne (de Jesus et al 2004b). If RESI is the dominating mechanism of NSDI one finds a maximum at zero momentum but still a broader distribution compared to SDI (Feuerstein et al 2001). In the case of 
$\mathrm{H}_{2}$ one has to consider the sum (or centre-of-mass) momentum of the two protons which plays the role of the recoil ion momentum in the atomic case and balances the sum momentum of the electrons. Figure 7 shows the protons sum momentum distributions for the CREI and NSDI channels for $25 \mathrm{fs}$ and $7 \mathrm{fs}$ laser pulses. In both cases one finds a significantly broader distribution for NSDI compared to CREI. A double-hump structure is not found in the recollision case providing additional evidence that RESI is the dominating mechanism as it has been recently discussed by Tong et al (2003). It can be noticed that the NSDI momentum distribution is slightly broadened for a few-cycle pulse while the SDI spectra have almost the same widths. Low statistics does not allow us to draw a conclusion whether this broadening indeed reflects some changes in the recollision dynamics. To clarify this point, kinematically complete experiments on rescattering-double ionization of $\mathrm{H}_{2}$ are under way.

\subsection{Dissociation in ultrashort pulses}

When the laser pulse duration is decreased from 25 to $10 \mathrm{fs}$, the most remarkable change in the low-energy part of the proton spectrum is the reversed branching ratio of $1 \omega$ and $2 \omega$ dissociation channels (Figure $3 a$ and $3 b$ ). Enhancement of the $1 \omega$ peak for tpulses with very short rise-times was discussed by Nomico et al (1997) in terms of "below-threshold" dissociation. Later, Frasinski et al (1999) experimentally observed the dynamical shift of this structure to higher energies with decreasing pulse duration, which was associated with the different speed of the release of the trapped wave packet at the falling edge of the pulse. For 10 and 7 fs pulses we also observe a slight shift of the position of the $1 \omega$ peak compared to $25 \mathrm{fs}$. However, it is not clear to which extent effects like vibrational trapping or "below-threshold" dissociation can contribute in our case of high intensities. In general, with decreasing temporal width 
of the pulse the shape of the dissociation part of the spectrum deviates more and more from the two-peak structure corresponding to the $1 \omega$ and $2 \omega$ dissociation channels (see curve 2 in Figure 3c). The reason for this, apart from the broadening of the photon spectrum, is the breakdown of the Floquet model for the dissociation which assumes a quasi-CW laser field. The propagation of the wavepackets on the lightinduced potential curves via avoided crossings (see Figure 1) into the dissociation continuum needs time. If the pulse duration becomes shorter than the time required to reach the level crossings, this mechanism does not work anymore, similar to the suppression of CREI discussed above. However, even in the limit of an infinitely short " $\delta$-like" pulse the molecule will still have a finite probability to dissociate, since the vertical (Franck-Condon) transition from $\mathrm{H}_{2}$ to $\mathrm{H}_{2}{ }^{+}$in the preceding single ionization gives rise to a small overlap of the initial nuclear wave function with the dissociation continuum of $\mathrm{H}_{2}^{+}$. The corresponding fragment energy distribution is compared to the experimental result for a 7 fs pulse in Figure 6. The best agreement is found for $0.8 \mathrm{PW} / \mathrm{cm}^{2}$ with a residual deviation most likely due to the finite pulse duration. This difference becomes stonger stronger at higher intensities in form of a partial reappearance of the two light-induced dissociation peaks since even weak post pulses may be strong enough to open the dissociation paths through the avoided crossings. (This can be confirmed by comparison of the curves 1 and 2 in Figure 3c). The lower intensity shows a remarkable lack of higher dissociation energies compared to the Franck-Condon distribution. This is most likely caused by the $R$-dependent ionization potential of $\mathrm{H}_{2}$ which leads to a strong suppression of the ionization probability at small $R$. As a consequence the Franck-Condon distribution is shifted to larger $R$ which reduces the number of more energetic fragments. Such an effect has been demonstrated in a recent joint experimental and theoretical work (Urbain et al 
2004) for the population of vibrational states of $\mathrm{H}_{2}{ }^{+}$in strong-field single ionization of $\mathrm{H}_{2}$.

The question remains whether all dissociation products are restricted to the lowenergy region. Figure 8 displays the proton energy spectra corresponding to the coincidence diagram in Figure $5 b$ and curve 1 in Figure $3 c$. The dashed line shows the energy distribution for the events where two protons are detected in coincidence and momentum conservation is fulfilled. This makes the separation of double ionization and dissociation events more reliable. The spectra are scaled in order to match the number of single proton events in the CREI region. Regions of the spectrum where random coincidences cannot be excluded are marked by shaded areas. We find a significant deficit of coincident events around 3 to $6 \mathrm{eV}$, i.e. in the region of SDI. This indicates indirectly a contribution of dissociation since neutral fragments are not detected. The origin of this energetic dissociation fragments remains unclear. One possible contribution can be given by the RESI mechanism for NSDI. In its second step the electron in the recollision-induced excited state is field-ionized. If the laser pulse duration is short enough even at high peak intensities some population in excited states may survive. Since all corresponding potential curves are repulsive, the electronically excited molecule will dissociate. Figure 1 shows that e.g. recollision population of the first excited state $2 \mathrm{p} \sigma_{\mathrm{u}}$ will lead to a total KER of 5 to $12 \mathrm{eV}$ (i.e. 3 to $6 \mathrm{eV}$ per proton) which is in good accordance with our experimental findings. Similar values are expected for the $2 \mathrm{p} \pi_{\mathrm{u}}$ state. There is an ongoing debate whether dissociation or $\mathrm{CE}$ is the dominating channel in the "molecular clock" scheme. Niikura et al 2003a,b attributed the main feature in the fragment spectrum around 6 $\mathrm{eV}$ to the dissociation of recollision-excited molecular ions. However, their noncoincident experiment could not distinguish reliably between dissociation and CE. 
More recently Tong et al 2003 demonstrated that both channels contribute and discussed in detail the noncoincident and coincident (Alnaser et al 2003) spectra. For the dissociation fragments a KER per particle of 2 to $7 \mathrm{eV}$ is calculated which is in reasonable agreement with our results. However, it seems unlikely that in our case recollision-induced dissociation is the dominant source of the high-energy dissociated protons since we do not restrict the solid angle in order to select the recollision contribution, as was done in the experiment of Alnaser et al (2003).

\section{Conclusions and outlook}

We have studied the dynamics of molecular hydrogen fragmentation by intense laser pulses of different durations. For 25 fs pulses we observed the spectra well-known from previous studies, with $1 \omega$ and $2 \omega$ dissociation peaks, a broad CREI structure and high-energy tail due to recollision-induced Coulomb explosion. When the pulse width is reduced, the two-peak dissociation structure in the proton kinetic energy spectra is smeared out, and for $7 \mathrm{fs}$ becomes closer to the Franck-Condon distribution. Coulomb explosion (CREI) contribution is suppressed, and "atomic-like" sequential double ionization which results in the production of the protons with higher kinetic energies starts to be distinguishable. The intensity dependence of this SDI is in a good qualitative agreement with the recent theoretical results (Tong and Lin 2004). Coincident measurements revealed the existence of a low-energy Coulomb explosion peak which for non-coincident data is hidden in the dissociation part. Experiments with different pulse shapes showed that this structure is most likely due to delayed ionization caused by the post pulses at large internuclear distances. This low-energy structure, as well as residual CREI contribution can be enhanced (see curve 1 in 
Figure 3c, Figure 5b and Figure8), if 7 fs pulses with higher pre and post pulses are used.

There are several questions that remain open. The low-energy CE peak seems to be well-localized. An interesting point is whether two double ionization peaks $1 \mathrm{n}$ Figure 8 (at $\sim 1.5$ and $2.5 \mathrm{eV}$ ) reflect two maxima in the R-dependence of the probability of enhanced ionization, that were predicted theoretically (see, e.g. Zuo and Bandrauk 1995) but never observed experimentally. To answer this question, pumpprobe experiments with few-cycle pulses are planned. Another problem concerns the observed deficit of coincidence events for proton kinetic energies of 3-6 eV. To clarify this point, more detailed coincident measurements with "clean" 7 fs pulses together with reliable time-dependent description of the fragmentation process are required. And, of course more information on the dynamics of different fragmentation channels can be obtained from the coincident electron spectra. First results for $30 \mathrm{fs}$ were reported earlier (Rottke et al 2002), and the experiments with few-cycle pulses are under way.

The authors are grateful to $\mathrm{H}$. Rottke and A. Voitkiv for valuable discussions.

\section{References}

Alnaser A S, Osipov T, Benis E P, Wech A, Shan B, Cocke C L, Tong X M and Lin C D 2003 Phys. Rev. Lett. 91163002

Bandrauk A D and Sink M L 1981 J. Chem. Phys. 741110

Bandrauk A D and Shon M H 2002 Phys Rev. A 66031401

Bucksbaum P H, Zavriyev A, Muller H G and Schumacher D W 1990 Phys. Rev. Lett. 641883 
Chelkovski S, Conjusteau A, Zuo T and Bandrauk A 1996 Phys. Rev. A 543235

Chelkovski S, Corkum P B and Bandrauk A 1999 Phys. Rev. Lett. 823416

Dundas D, McCann J F, Parker J S and Taylor K T 2000 J. Phys. B 333261

Feuerstein B, Moshammer R, Fischer D, Dorn A, Schröter C D, Deipenwisch J, Crespo Lopez-Urrutia J R, Höhr C, Neumayer P, Ullrich J, Rottke H, Trump C, Wittmann M, Korn G and Sandner W 2001 Phys. Rev. Lett. 87043003

Feuerstein B and Thumm U 2003 Phys. Rev. A 67043405

Frasinski L J, Posthumus J H, Plumridge J, Codling K, Taday P F and Langley A J 1999 Phys. Rev. Lett. 833625

Gibson G N, Li M, Guo C and Neira J 1997 Phys. Rev. Lett. 792022

Giusti-Suzor A, He X, Atabek O and Mies F H 1990 Phys. Rev. Lett. 64515

Giusti-Suzor A, Mies F H, DiMauro L f, Charron E and Yang B 1995 J. Phys. B 28 309

Harumiya K, Kono H,Fujimura Y, Kawata I and Bandrauk A 2002 Phys. Rev. A 66 043403

de Jesus V L B, Rudenko A, Feuerstein B, Zrost K, Schröter C D, Moshammer R and Ullrich J 2004a J. J. Electron Spectrosc. Relat. Phenom. (in press)

de Jesus V L B, Feuerstein B, Zrost K, Fischer D, Rudenko A, Afaneh F, Schröter C D, Moshammer R and Ullrich J 2004b J. Phys. B 37 L161

Niikura H, Légaré F, Hasbani R, Bandrauk A D, Ivanov M Y, Villeneuve D M and Corkum P B 2002 Nature 417917

Niikura H, Légaré F, Hasbani R, Ivanov M Y, Villeneuve D M and Corkum P B 2003 Nature $\mathbf{4 2 1} 826$

Numico R, Keller A and Atabek O 1997 Phys. Rev. A 56772 
Osipov T, Alnaser A S, Voss S, Prior M H, Weber T, Jagutzki O, Schmidt L,

Schmidt-Bocking H, Dorner R, Landers A, Wells E, Shan B, Maharjan C, Ullrich

B, Ranitovic P, Tong X M, Lin C D and Cocke C L 2004 J Mod. Opt. (in press)

Plummer M and McCann J F 1996 J. Phys. B 294625

Posthumus J H, Plumridge J, Frasinski L J, Codling K, Divall E J, Langley A J and Taday P F 2000 J. Phys. B 33 L563

Posthumus J H 2004 Rep. Prog. Phys. 67623

Rottke H, Trump C, Wittmann M, Korn G, Sandner W, Moshammer R, Dorn A, Schröter C D, Fischer D, Crespo López-Urrutia J R, Neumayer P, Deipenwisch J, Höhr C, Feuerstein B, and Ullrich J 2002 Phys. Rev. Lett. 89013001

Saenz A 2002a Phys. Rev. A 66063407

Saenz A 2002b Phys. Rev. A 66063408

Sakai H, Larsen J J, Wendt-Larsen I, Olesen J, Corkum P B and Stapelfeldt H 2003 Phys. Rev. A 67063404

Sändig K, Figger H and Hänsch T W 2000 Phys. Rev. Lett. 854876

Shellhammer C, Schyja V, Hielscher A and Helm H 2000 Laser and Particle Beams 18443

Shirley J H 1965 Phys. Rev. 138 B979

Staudte A, Cocke C L, Prior M H, Belkacem A, Ray C, Chong H W, Glover T E, Schoenlein R W and Saalmann U 2002 Phys. Rev. A 65020703

Thomson M R, Thomas M K, Taday P F, J, Posthumus J H, Langley A, Frasinski L J and Codling K J 1997 J. Phys. B 305755

Tong X M, Zhao Z X and Lin C D 2003 Phys. Rev. A 68043412

Tong X M and Lin C D 2004 Phys. Rev. A 70023406 
Ullrich J, Moshammer R, Dorn A, Dörner R, Schmidt L Ph H and Schmidt-Böcking H 2003 Rep. Prog. Phys. 661463

Urbain X, Fabre B, Staicu-Casagrande E M, de Ruette N, Andrianarijaona V M, Jureta J, Posthumus J H, Saenz A, Baldit E and Cornaggia C 2004 Phys. Rev. Lett. 92163004

Walsh T D G, Ilkow F A and Chin S I 1997 J. Phys. B 302167

Walsh T D G, Ilkow F A, Chin S I, Châteauneuf F, Nguyen-Dang T T, Chelkovski S, Bandrauk A and Atabek O 1998 Phys. Rev. A 583922

Weber Th, Weckenbrock M, Staudte A, Spielberger L, Jagutzki O, Mergel V, Afaneh F, Urbasch G, Vollmer M, Giessen H and Dörner R 2000 J. Phys. B 33 L127

Yu H, Zuo T and Bandrauk A 1996 Phys. Rev. A 543290

Zavriyev A, Bucksbaum P H, Squier J and Saline F 1993 Phys. Rev. Lett. 701077

Zuo T and Bandrauk A 1995 Phys. Rev. A 52 R2511 


\section{Figure legends:}

Figure 1. Potential curves of two lowest electronic states of $\mathrm{H}_{2}{ }^{+}$dressed with $\mathrm{n}(\mathrm{n}=$ $1,2,3$ ) photons. Arrows indicate different fragmentation pathways.

Figure $2 . \mathrm{H}_{2}$ fragmentation spectra with 25 fs pulses at $0.2 \mathrm{PW} / \mathrm{cm}^{2}$.

(a) Typical time-of-flight ion spectra. The dashed vertical line indicates time-of-flight of the protons with zero momentum along the field polarization direction. (b) Solid line: corresponding proton energy spectrum for the "forward" protons (see text). Dashed line: the data obtained with circularly polarized light. The curve is normalized to fit the $2 \omega$ peak for linear polarization. In circular case the intensity was 2 times lower.

Figure 3. Evolution of the proton time-of-flight spectra with the pulse duration.

(a) 25 fs pulses with the intensities of $0.2,0.3$ and $0.5 \mathrm{PW} / \mathrm{cm}^{2}$ for the lower, middle and upper curve, respectively. The curves are separated in vertical direction for visual convenience. (b) $10 \mathrm{fs}, 0.5 \mathrm{PW} / \mathrm{cm}^{2}$. (c) $7 \mathrm{fs}, 0.8 \mathrm{PW} / \mathrm{cm}^{2}$. Curves 1 and 2 were obtained with the pulses of different shape (see text and Figure 4).

Figure 4. Characteristics of two laser pulses corresponding to the results of Figure 3c. (a), (d) Measured frequency spectra. (b), (e): Fourier limit for the intensity profile. (c), (f) Measured $2^{\text {nd }}$ order autocorrelation. Panels (a)-(c) and (d)-(f) correspond to the curves 1 and 2 in Figure 3c, respectively.

Figure 5. Coincidence diagram of the proton longitudinal momenta.

The momentum of the protons detected on the detector as a first hit $\left(\mathrm{P}_{\mathbf{1}}{ }^{\|}\right)$is plotted versus the momentum of the second one $\left(\mathrm{P}_{2}{ }^{\|}\right)$. The momentum distribution is recalculated from the ion time-of-flight and thus contains the contribution of $\mathrm{H}_{2}^{+}$ions 
(a peak above 30 a.u.) which does not reflect their momenta. The real coincidences lay near the diagonal $\mathrm{P}_{\mathbf{1}} \|=-\mathrm{P}_{2}{ }_{2}^{\|}$due to momentum conservation.

(a) $25 \mathrm{fs}, 0.5 \mathrm{PW} / \mathrm{cm}^{2}$. (b) $7 \mathrm{fs}, 0.8 \mathrm{PW} / \mathrm{cm}^{2}$. The diagram corresponds to the curve 1 in Figure 3c and panels (a)-(c) in Figure 4. For discrete Coulomb explosion peaks the estimated values of the internuclear distances $\mathrm{R}$ are indicated.

Figure 6. Proton kinetic energy distributions obtained with 7 fs laser pulse.

The curves are separated in vertical direction for visual convenience. The intensities from up to down: $2.0 \mathrm{PW} / \mathrm{cm}^{2}, 1.4 \mathrm{PW} / \mathrm{cm}^{2}, 0.8 \mathrm{PW} / \mathrm{cm}^{2}, 0.5 \mathrm{PW} / \mathrm{cm}^{2}, 0.3 \mathrm{PW} / \mathrm{cm}^{2}$, $0.2 \mathrm{PW} / \mathrm{cm}^{2}$. The data were obtained with the "clean" pulses (the curve for 0.8 $\mathrm{PW} / \mathrm{cm}^{2}$ corresponds to the curve 2 in Figure $3 \mathrm{c}$ and panels (d)-(f) in Figure 4). Vertical arrows indicate positions of SDI maxima. Dashed lines show a FranckCondon distribution (see text).

Figure 7. Sum longitudinal momentum of two coincident protons created by sequential (a), (c) and non-sequential (b), (d) double ionization of $\mathrm{H}_{2}$ at $0.5 \mathrm{PW} / \mathrm{cm}^{2}$. (a), (b): 25fs. (c), (d): $7 \mathrm{fs}$.

Figure 8. Kinetic energy spectra of coincident and non-coincident protons. The set of data is the same as for the Figure $5 b$ and curve 1 in Figure $3 c$. Solid line: all events. Dashed line: events, detected in coincidence. Shaded areas mark the regions, where false coincidences cannot be excluded. 


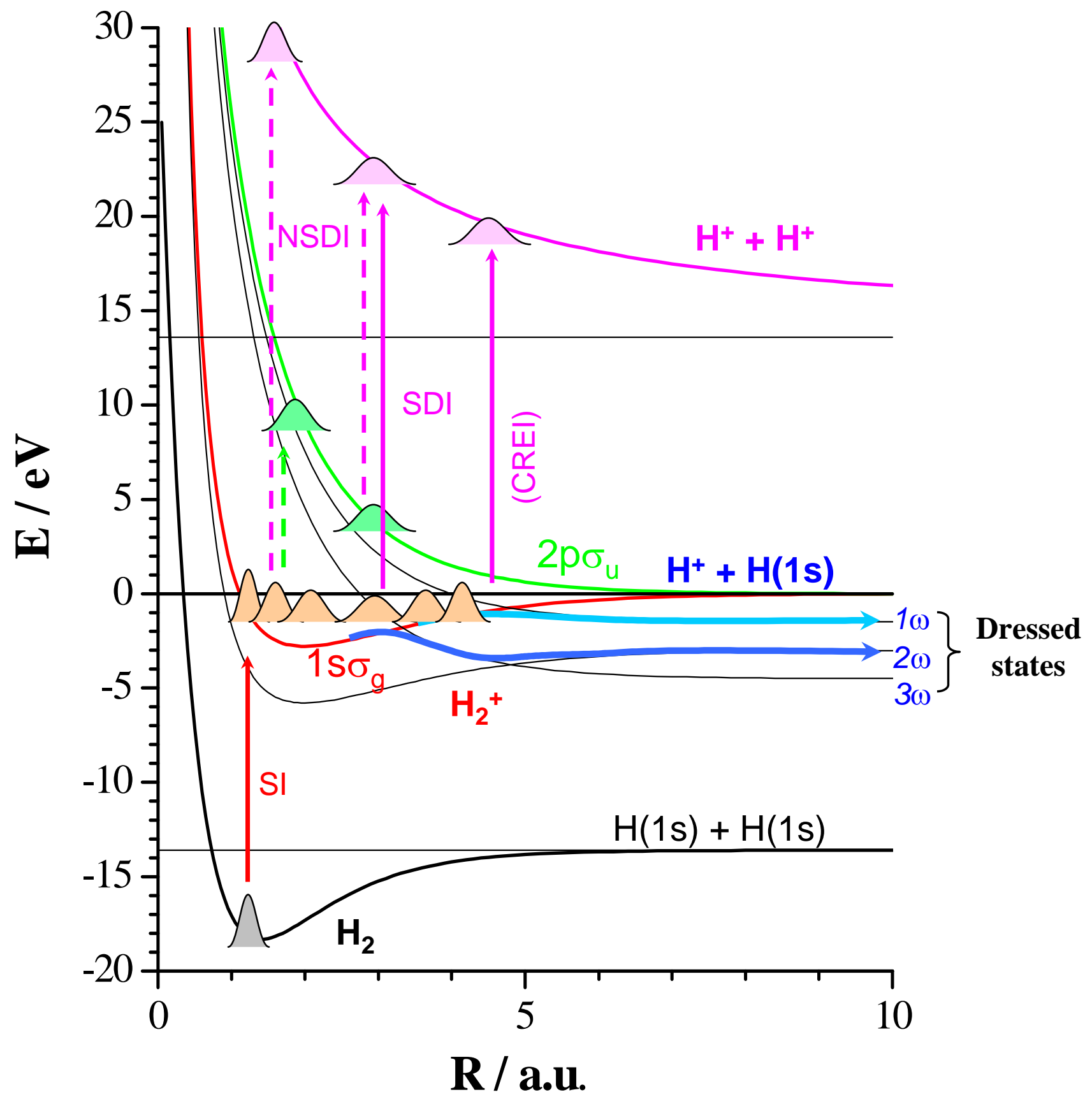

Figure 1 (color online) 

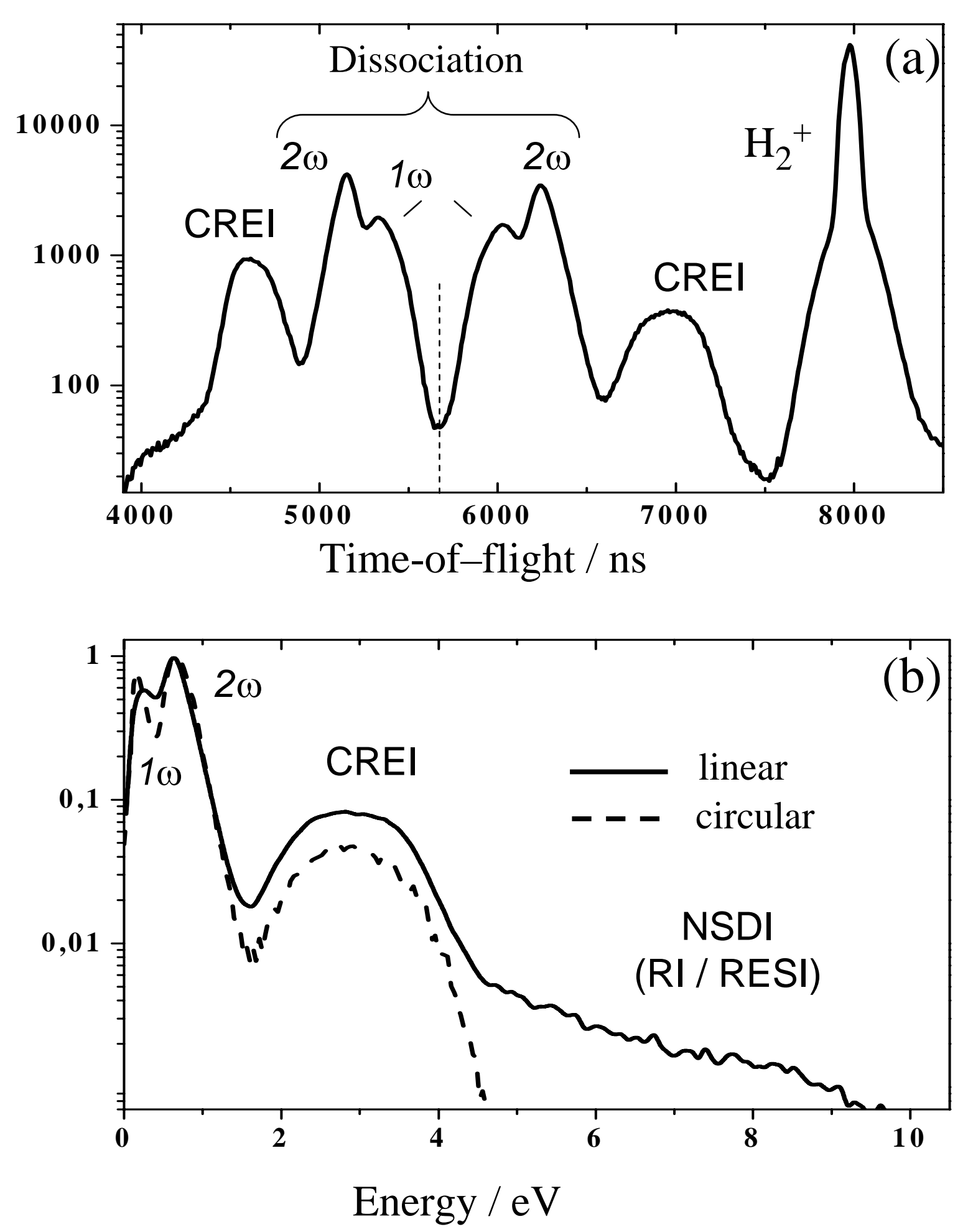

Figure 2 


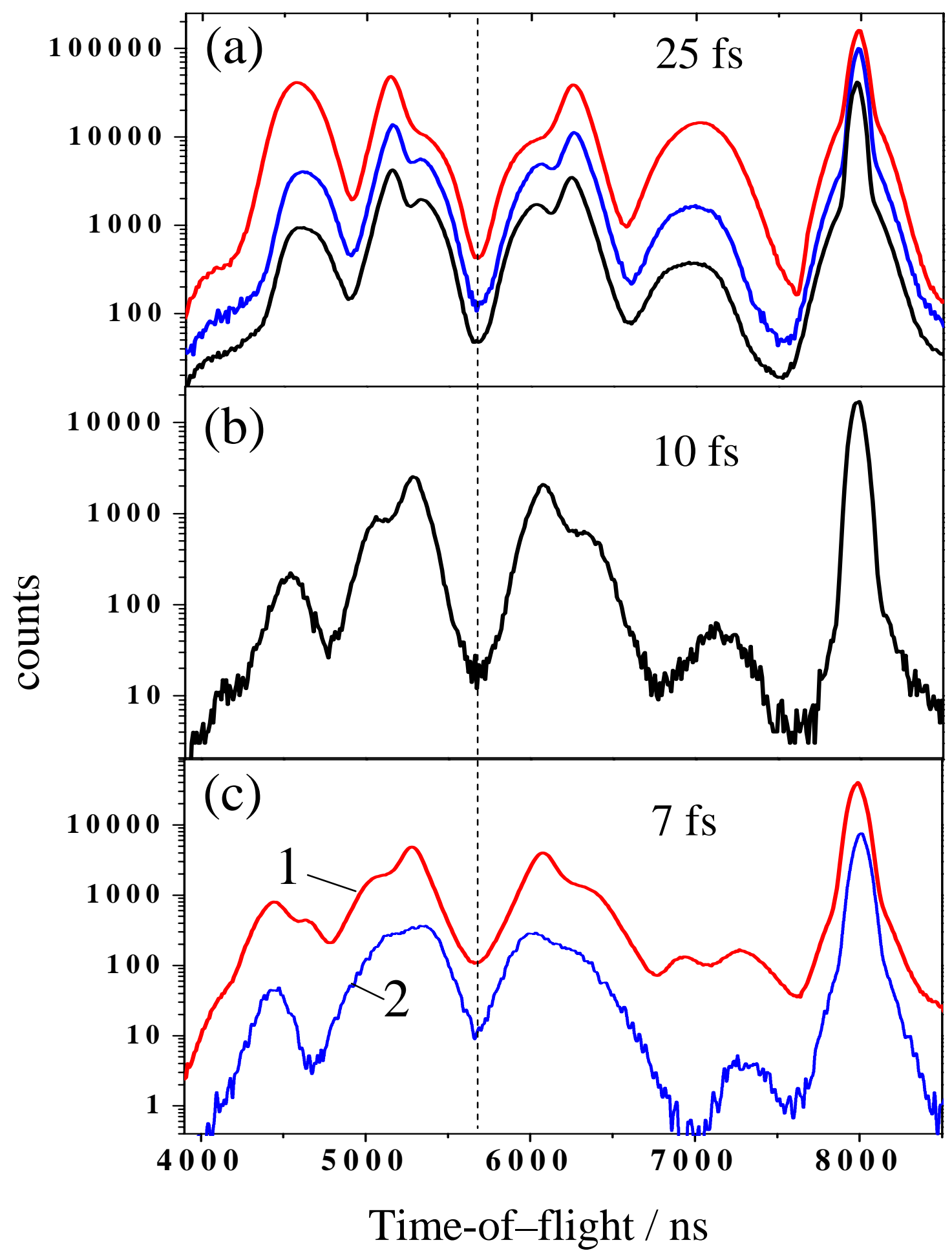

Figure 3 (color online) 

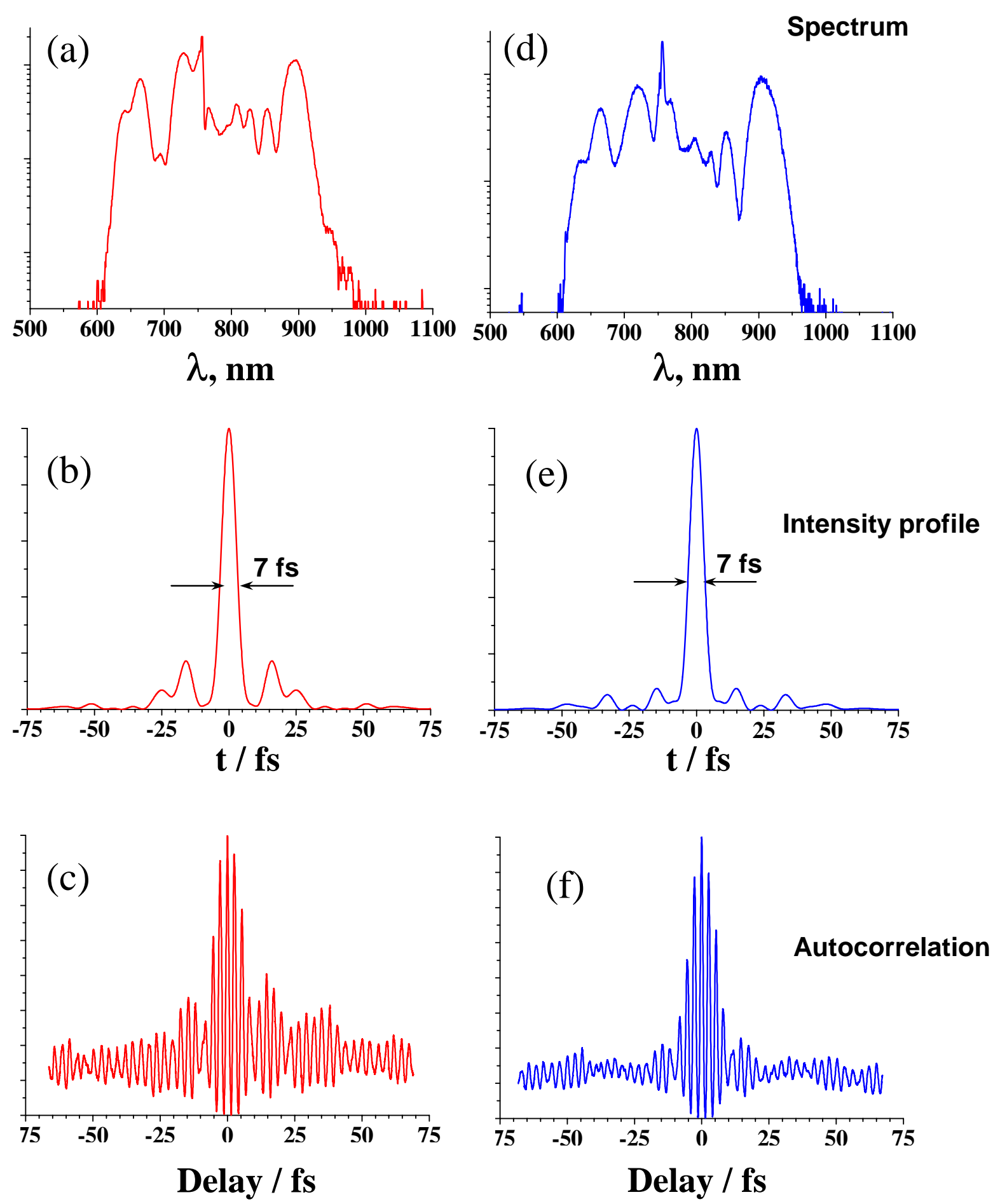

Figure 4 (color online) 


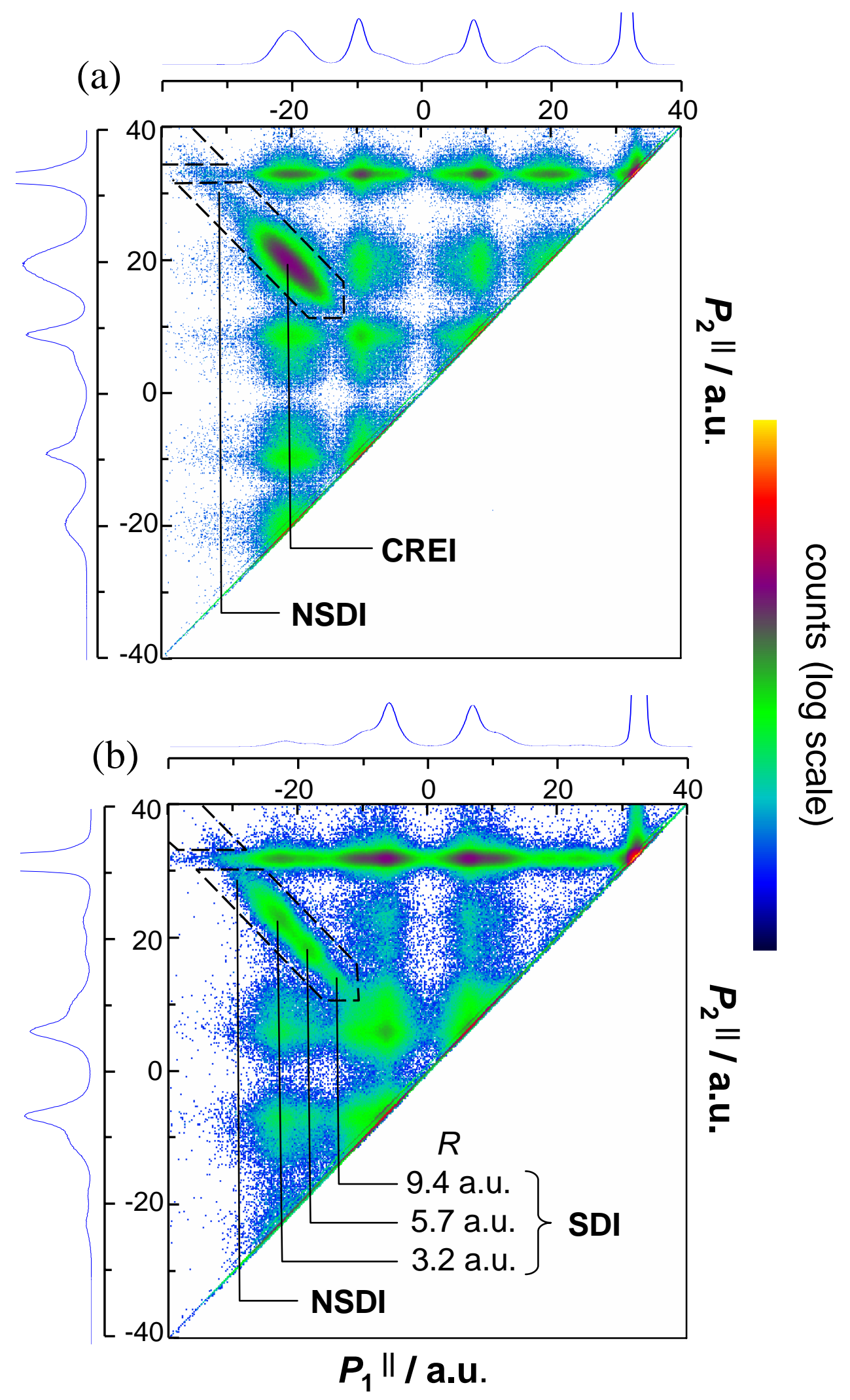

Figure 5 (color online) 


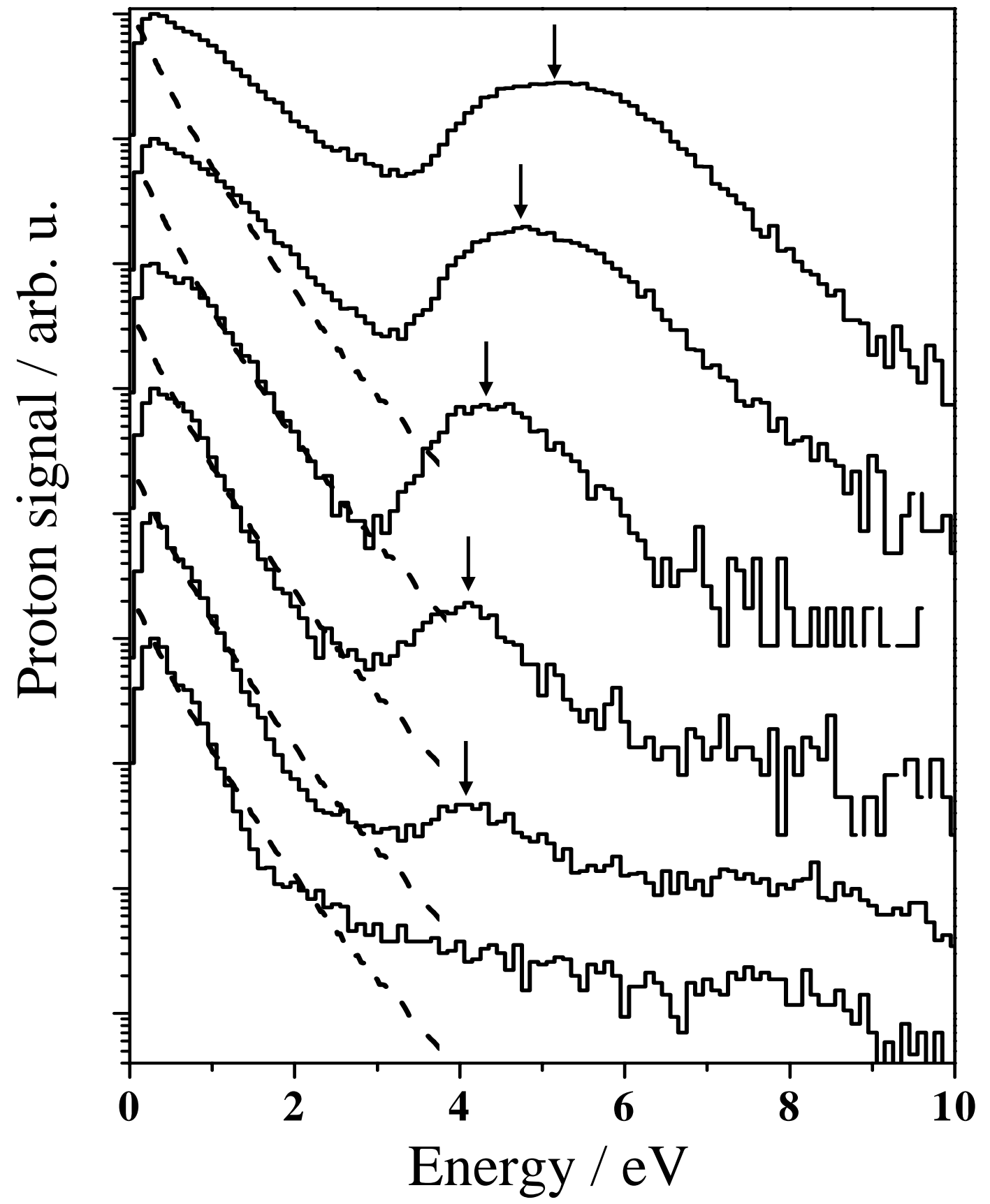

Figure 6 
25 fs

7 fs
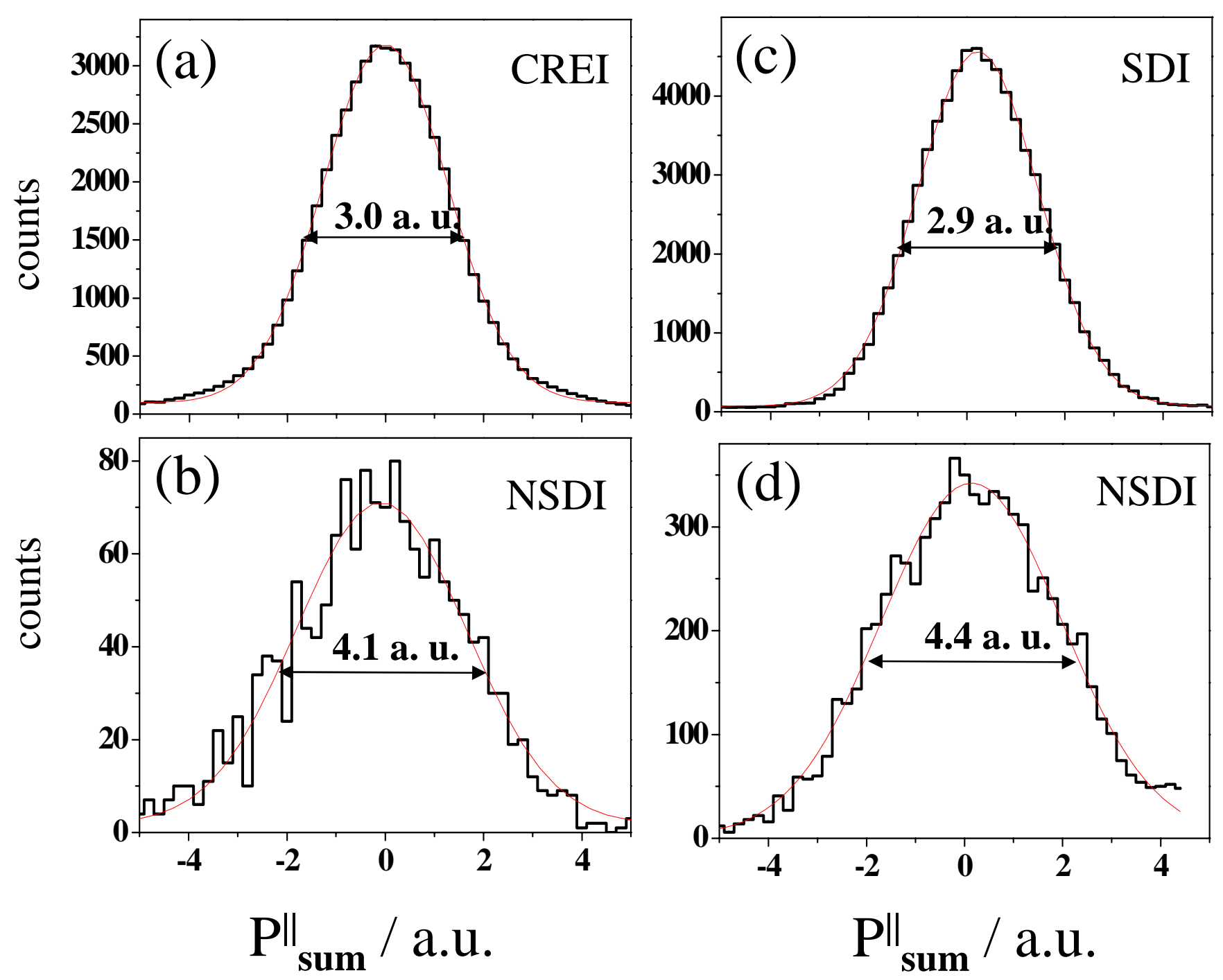

Figure 7 


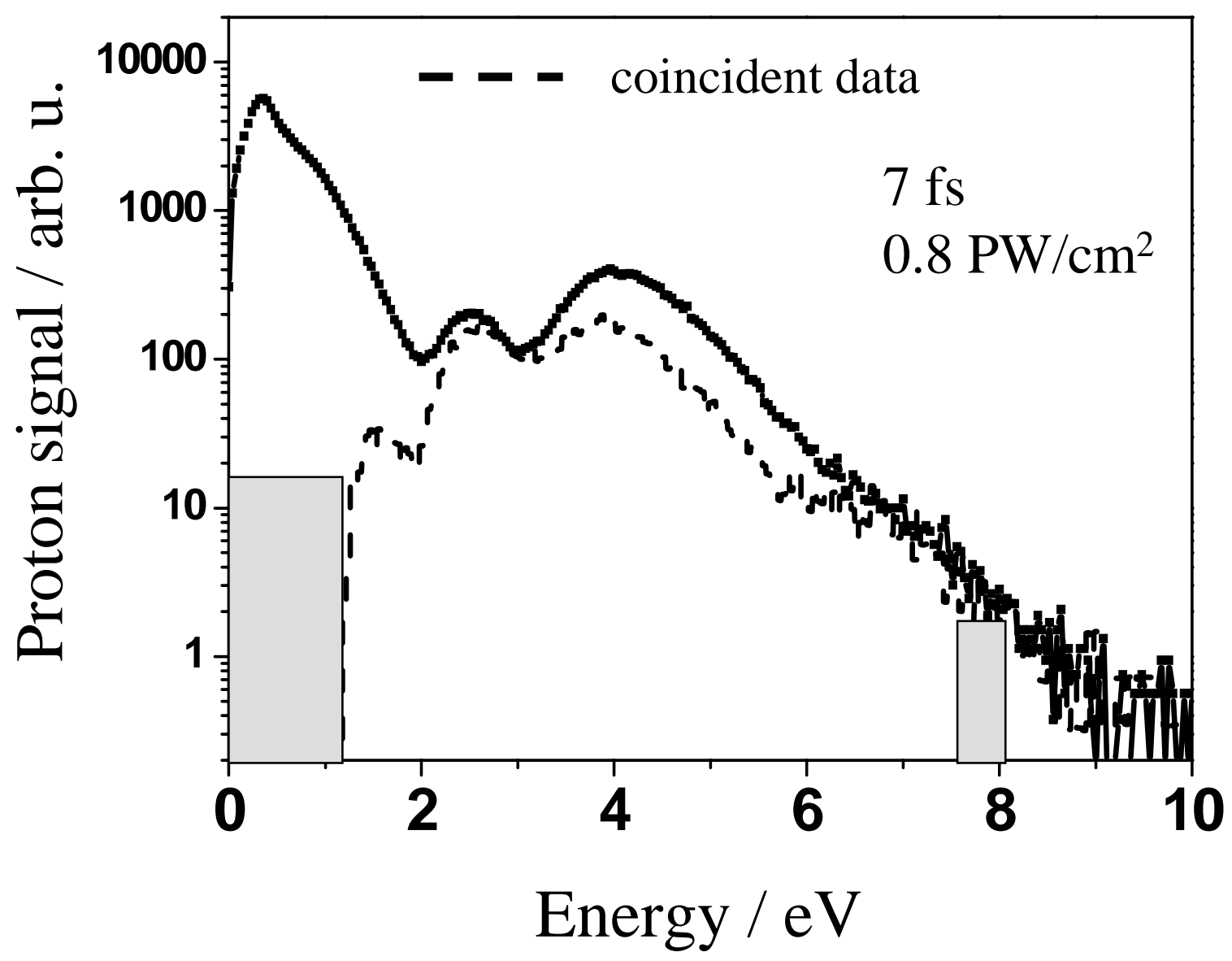

Figure 8 